\title{
Surface Modification of Space Exposed Materials Induced by Low Energetic Proton Irradiation
}

\author{
Denise Keil $^{1}$ (D) . Frederic Seiz ${ }^{1}$ - Nils Bartels ${ }^{1}$. Wolfgang Riede ${ }^{1}$. \\ Maciej Sznajder ${ }^{2,3}$. Thomas Renger ${ }^{2}$. Tom Spröwitz ${ }^{2}$
}

Accepted: 8 July 2021 / Published online: 17 August 2021

(C) The Author(s) 2021

\begin{abstract}
The aim of this paper is to verify if commonly occurring space debris materials change their reflectivity and morphology after being exposed to low energy protons. Therefore, a set of six different materials frequently used in spacecraft engineering was irradiated with low energy $(100 \mathrm{keV})$ protons to simulate the aging of their surfaces due to space radiation in low Earth orbit (LEO). A microscopic and spectroscopic analysis of the irradiated samples reveals that the tested materials containing organic polymers (Polytetrafluoroethylene (PTFE) and carbon fiber reinforced plastic (CFRP)) show changes in surface morphology. Metallic surfaces did not show surface modifications but we found changes in the reflectivity of coated polyimide sheets, like used in Multi Layer Insulation blankets, during and after proton irradiation. Our results show that space materials exhibit significant changes after irradiation equivalent to the dose accumulated after 100 years in LEO. This knowledge is highly relevant for the interpretation of optical data related to the observation of space debris as well as to studies about laser-matter interaction for laser-based debris removal.
\end{abstract}

Keywords Space aging $\cdot$ Space debris $\cdot$ Characterization $\cdot$ Complex irradiation facility $\cdot$ Protons

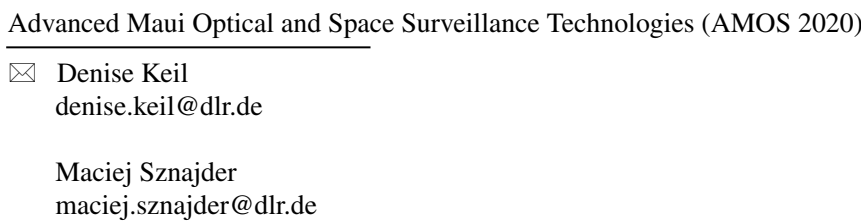

1 Institute of Technical Physics, German Aerospace Center DLR, Pfaffenwaldring 38 - 40, 70569 Stuttgart, Germany

2 Institute of Space Systems, German Aerospace Center DLR, Robert-Hooke-Strasse 7, 28359 Bremen, Germany

3 MAPEX Center for Materials and Processes, University of Bremen, Bibliothekstr. 1, 28359 Bremen, Germany 


\section{Introduction}

Although since 2007 an increasing number of space missions follow the agreement of deorbiting satellites at their end of life [1], satellite operators have to deal with an increasing amount of space debris [2]. In case of potential collisions, operators can react by initiating collision avoidance maneuvers. Between the years 2005 to 2014, the number of these maneuvers has increased by a factor of eight [3]. Unfortunately, they are quite costly. Most debris fragments smaller than $10 \mathrm{~cm}$ are not detected and catalogued and thus cannot be avoided [4]. To improve this situation, space organizations like NASA, ESA, and JAXA are working on data bases cataloging space debris. Even for a scenario in which $90 \%$ of all space missions follow the mitigation standards, collisions of space debris are expected to lead to an increase in the space debris population in low Earth orbit (LEO) by $30 \%$ over the next 200 years [5]. The situation becomes even worse considering currently planned and ongoing mega constellation projects. Here an increase up to $590 \%$ until 2215 of debris particles larger $10 \mathrm{~cm}$ is predicted [6]. This suggests that active strategies for debris removal will become necessary to preserve the near Earth environment for future space missions.

An approach, for gathering additional information about the space debris environment in the LEO is extracting data from debris-reflected sunlight with telescopes, so called light curves [7]. These light curves are often measured with spectral or polarization sensitive detectors. Results are then evaluated with computer simulations to extract more details of space objects, such as their rotational period, their shape or their surface materials [8-11]. As a prerequisite for these simulations, it is necessary to provide the computer models with parameters for the reflectivity of different space materials, often resolved with respect to the incidence and scattered angle (socalled bidirectional reflectance distribution function, BRDF) and as a function of polarization and wavelength [9].

While this is already a difficult task in itself, a major additional challenge is that it can be insufficient to measure the reflectivity of space materials on Earth, since material properties might change during the exposure to the space environment. Various studies have been performed investigating surface degradation due to UV light [12], atomic oxygen [13], and particle irradiation [14, 15]. The results of these studies consistently show that the space environment can induce significant changes to optical and mechanical parameters. During the mission MISSE-6 (Materials International Space Station Experiment) [13], a set of a 168 materials was exposed for 18 months on the exterior of the International Space Station (ISS). It should, however, be noted that the space environment at the ISS (ca. $400 \mathrm{~km}$ orbital height) is dominated by atomic oxygen and UV solar radiation. The major space traffic in LEO is distributed to higher orbits, where three major peaks can be pointed out: one at roughly $500 \mathrm{~km}$ SSO (Sun Synchronous Orbit), a brought one in the region between 780 to $880 \mathrm{~km} \mathrm{SSO}$ and a third sharp one at $990 \mathrm{~km}$ and $83^{\circ}$ inclination $[6,16,17]$.

Rarely investigated are the changes of material properties due to low energetic proton and electron irradiation as encountered in the inner van Allen belt, which reaches from 0.2 to 2 Earth radii (L-Shells: $\mathrm{L}=1$ to $\mathrm{L}=3$ ) [18] and therefore is intersecting with the higher LEO orbits. This radiation belt is dominated by positively charged 
particles (protons) originating from the sun with a kinetic energy in the range of several keV up to $400 \mathrm{MeV}$ [19].

In this work we study the degradation of several materials due to energetic protons as encountered in the inner van Allen belt. A focus is on low energetic particles (100 $\mathrm{keV}$ ) since these particles are known to cause most severe surface modifications as they are stopped near the surface of the spacecraft material [20]. The goal of this work is to conclude how and which material properties, such as the surface morphology and reflectivity, are affected by the proton irradiation.

\section{Methods}

To investigate the effects of low energetic particle irradiation on material surfaces, we performed space aging experiments at the Complex Irradiation Facility (CIF) at the German Aerospace Center (DLR) in Bremen. Here, the main focus was on proton irradiation. The samples were investigated before and after radiation with incident light- and scanning electron microscopy, in order to detect possible changes to the surface morphology. We also performed in-situ measurements of the reflectivity at $632 \mathrm{~nm}$ using a measurement system, which we call LAMBDA (Laser cAMera alBeDo Analyzer). In the following, we briefly introduce the applied systems.

\section{CIF - Complex Irradiation Facility}

The CIF is an ultra-high vacuum system designed to experimentally simulate low energetic corpuscular and electromagnetic radiation and its impact on spacecraft material surfaces. The facility is equipped with three light sources and two particle accelerators providing protons and electrons, see Fig. 1. The radiation sources can operate separately or combined [21]. The light sources are an Argon-VUV-source, a Deuterium lamp, and a solar simulator equipped with a Xenon arc lamp, which can simulate the solar irradiance up to several solar constants. Accelerators produce monoenergetic protons and electrons in an energy range from 1 to $10 \mathrm{keV}$ at 1 to $100 \mathrm{nA}$ and 10 to $100 \mathrm{keV}$ with a current up to $100 \mu \mathrm{A}$. Both species are accelerated separately and then magnetically deflected to a common beam line which ends at the irradiation chamber where specimens under study are located at a temperaturecontrolled sample station. The facility is intentionally designed for the investigation of radiation effects on material surfaces [21, 22]. It has proven its value already in other research projects, mainly concerning deorbit sails and solar sailing [23].

\section{LAMBDA - Laser CAMera alBeDo Analyzer}

LAMBDA is a device we specifically designed for this work. It allows for an in-situ monitoring of the relative reflectivity at a wavelength of $632 \mathrm{~nm}$ under a given angle of material surfaces during the irradiation in the CIF. The system consists of a HeNeLaser, Canon EOS 5D Mark IV camera, and a beam expanding lens system, which is arranged as depicted in Fig. 2. Due to the geometry of the irradiation chamber, the laser beam, the camera axis and the proton beam are arranged under $30^{\circ}$ to each other. 


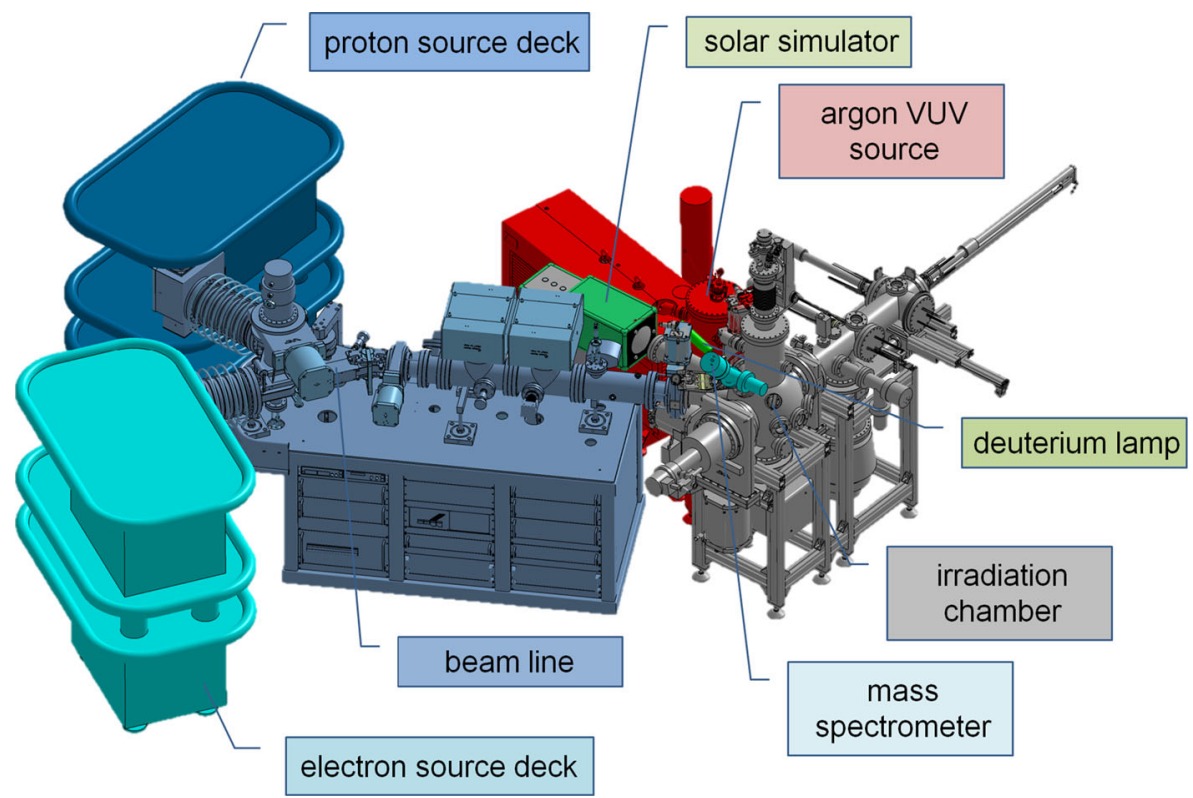

Fig. 1 Schematic of the complex irradiation facility

The measurement principle works as follows: red laser light is emitted by a low power HeNe-Laser. The expanded beam spot illuminates the samples inside the UHV-chamber while they are under particle irradiation. Since the reflection is

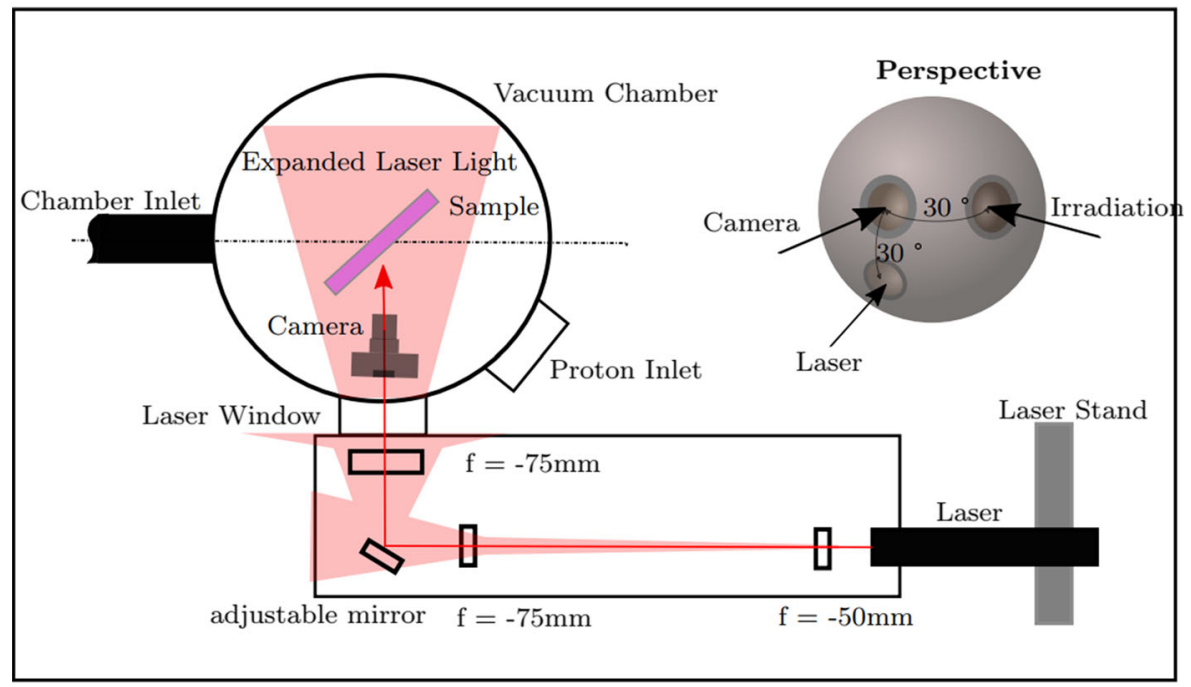

Fig. 2 Schematic principle of LAMBDA 
depending on the condition of a surface, the reflectivity per spatial angle is changing. This change is detected by the camera. Calculating the average value of a related pixel region gives information about the reflected light which is directed towards the camera. The charged particle irradiation time can be related to an orbit equivalent time. This approach allows to quantify how much a surface degenerates depending on orbit and time.

\section{Microscopes}

The microscopic inspection of the optical surfaces before and after irradiation in the CIF was performed with an incident light microscope, which is a Keyence Digital Microscope Type VHX-7000 Series. We took an stitch image of the overall sample surface before and after the irradiation as well as detail images of areas where predamages were existing. The dimensions and appearance of predamages were compared in terms of growth due to the exhibition to protons. Furthermore, we use a scanning electron microscope (SEM, Zeiss type EVO MA 10) to detect surface modifications at high magnification. Here we investigated a set of reference samples and compared their surface structure to the aged ones.

\section{Spectral Analysis}

A Fourier-Transform InfraRed Spectrometer (FTIR) Bruker Vertex70v has been used to analyze the reflectance spectra of the unirradiated reference samples and the irradiated samples in the wavelength range from $0.4 \mu \mathrm{m}$ to $10 \mu \mathrm{m}$. The visible range between $0.4 \mu \mathrm{m}$ and $1.0 \mu \mathrm{m}$ was measured using a tungsten light source combined with a silicon diode and the range $1.2 \mu \mathrm{m}$ to $10 \mu \mathrm{m}$ with the internal Globar light source and a room temperature DLaTGS (Deuterated Lanthanum $\alpha$ Alanine doped TriGlycine Sulphate) diode. As a reference mirror for reflectance measurements, a copper mirror was applied. The copper's spectrum was removed during post-processing. Here, we calculated the reflectivity of copper using the software "SCOUT" [24] and divided all data by the reflectivity of copper in order to get the absolute reflectivity of each sample. A measurement of the real copper mirror was taken to confirm its behavior over the full wavelength range.

\section{Experiment}

\section{Samples}

Figure 3 shows the sample holder used for the irradiation in the CIF. It carries 6 samples with the size of $21 \times 21 \mathrm{~mm}^{2}$ each. The samples, which are CFRP, Aluminum, ITO coated aluminized Polyimide Foil (MLI), AcktarBlack coated aluminum foil, Titanium, and PTFE, are listed in Table 1. These materials are frequently used in spacecraft engineering and represent different material groups. 


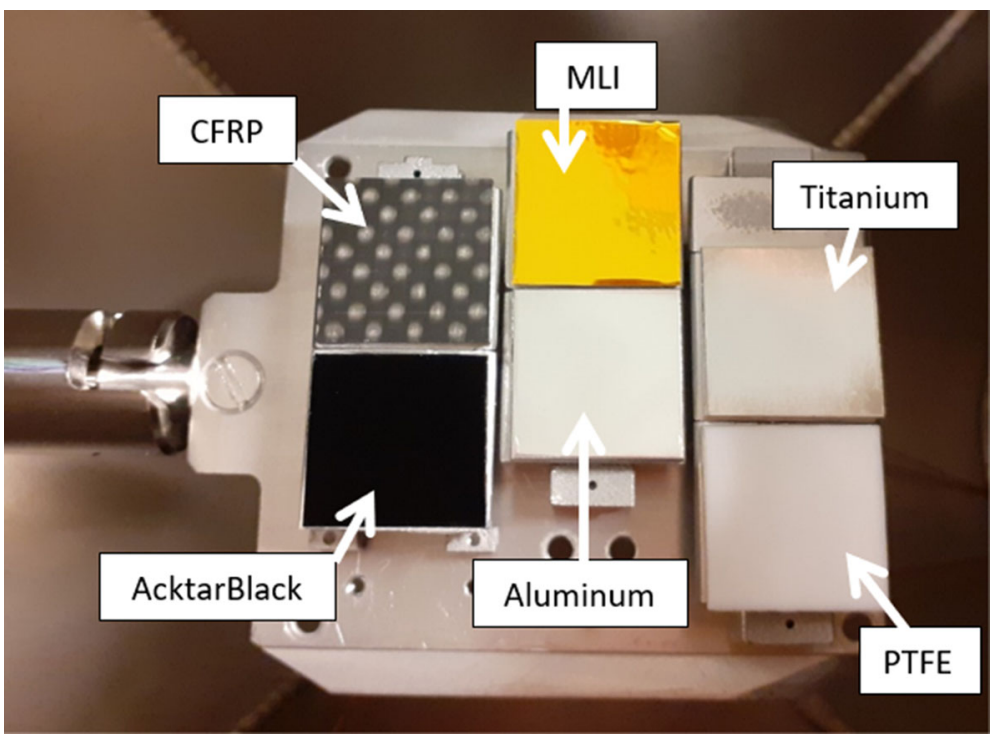

Fig. 3 Sample set

\section{Irradiation Parameters}

We decided to perform our irradiation tests using an irradiation dose representative for an orbital height of $800 \mathrm{~km}$, as this is where a majority of objects in low Earth orbit are resident [17]. The most populated inclination at this orbital height is the

Table 1 Properties of material choice

\begin{tabular}{|c|c|c|}
\hline Material & Description & Category \\
\hline $\begin{array}{l}\text { Carbon Fiber Reinforced } \\
\text { Plastic (CFRP) }\end{array}$ & $\begin{array}{l}\text { Fiber Fabric (canvas) with } \\
\text { Epoxy resin, } 1 \mathrm{~mm} \text { thickness }\end{array}$ & Polymer, black \\
\hline AcktarBlack & $\begin{array}{l}\text { Type: Spectral BlackTM, } \\
\text { Layer thickness: } 3-7 \mu \mathrm{m} \text { on } \\
125 \mu \mathrm{m} \text { Aluminum foil, } 99.5- \\
99.7 \% \text { absorbtivity for wave- } \\
\text { length range } 0.3-14 \mu \mathrm{m}\end{array}$ & Metal, black surface \\
\hline Coated polyimide & $\begin{array}{l}\text { Coating: ITO - Polyimide - } \\
\text { Aluminum, Type: Sheldahl No } \\
\text { 146631, typically applied in } \\
\text { Multilayer Insulation }\end{array}$ & $\begin{array}{l}\text { Polymer, polished metal } \\
\text { surface (golden) }\end{array}$ \\
\hline Aluminum 6082 & $\begin{array}{l}2 \mathrm{~mm} \text { thick material with } \\
\text { polished surface }\end{array}$ & Metal, polished metals surface \\
\hline Titan & $\begin{array}{l}0.8 \mathrm{~mm} \text { thick material with } \\
\text { polished surface }\end{array}$ & Metal, polished metals surface \\
\hline $\begin{array}{l}\text { Polytetrafluorethylene } \\
\text { (PTFE, Teflon) }\end{array}$ & $\begin{array}{l}\text { Commercially available } \\
\text { material with } 1 \mathrm{~mm} \text { thickness }\end{array}$ & Polymer, white \\
\hline
\end{tabular}


sun synchronous orbit (SSO). In this orbit, the amount of atomic oxygen can be neglected, whereas the density of charged particles (protons and electrons) is rather high. This density of protons $\left(\mathrm{p}^{+}\right)$and electrons $\left(\mathrm{e}^{-}\right)$was estimated using SPENVIS (SPace ENVironment Information System), where AE8 and AP8 models have been used to calculate the electron and proton fluxes, respectively [25]. In Fig. 4 we display the fluence over the particle energy. The fluence sums up all particles per centimeter square, which are on a chosen energy level and above. We calculated both extreme cases during the sun cycle, which is the solar maximum (solid lines), where the sun peaks in giant particle and mass eruptions, and the solar minimum (dotted lines), where the sun activity is low and calm. We found that the minimum and maximum fluencies of electrons (blue lines) and protons (black lines) are on a comparable level during the sun cycle. The largest proton fluence equals to $2.2 \times 10^{13} \mathrm{p}^{+} \mathrm{cm}^{-2}$ and corresponds to proton energy of $100 \mathrm{keV}$. The CIF has ability to produce protons with maximum kinetic energy of $100 \mathrm{keV}$. Exposed area is $6.2 \times 7.3 \mathrm{~cm}^{2}$. If one takes a current of $0.1 \mu \mathrm{A}\left(6.24 \times 10^{11} \mathrm{p}^{+} \mathrm{s}^{-1}\right)$, then the flux over the area is $1.38 \times 10^{10} \mathrm{p}^{+}$ $\mathrm{cm}^{-2} \mathrm{~s}^{-1}$. Under these conditions, 100 years in LEO's $800 \mathrm{~km}$ SSO orbit are simulated in 26.6 minutes.

In orbit, any material will encounter a continuous spectrum of particles with different kinetic energy over a period of several years. As opposed to this, the CIF generates mono-energetic particle radiation and the fluence will be applied over short time. As we are interested in changes to the optical surface, we decided - in an initial step - to irradiate samples with protons of a kinetic energy of $100 \mathrm{keV}$, since there are reliable models to estimate the irradiation dose. Since the penetration depth lies between 0.6 and $1.1 \mu \mathrm{m}$ in the investigated materials, depending on the material properties, it is likely to induce surface modifications at these conditions. The irradiation parameters were chosen to $100 \mathrm{nA}$ at $100 \mathrm{keV}$ for $26.6 \mathrm{~min}$, which represents an in-orbit duration of 100 years at $800 \mathrm{~km} \mathrm{SSO}$. The expectable errors are listed in Table 2 below.

Considering the worst cases we irradiated within a time range between 95.5 and 104.5 years. The greatest impact is the fluctuation in the proton current. Since it is

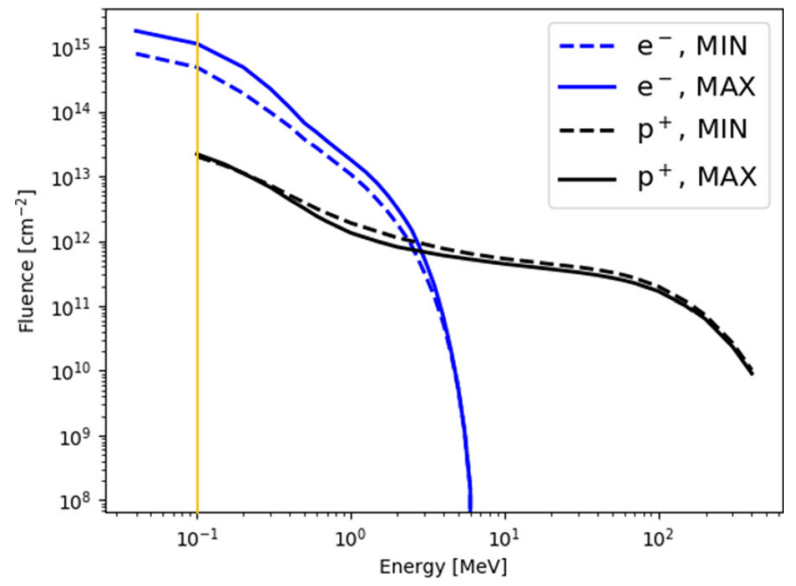

Fig. 4 Electron and proton fluence corresponding to 100 years in the $800 \mathrm{~km} \mathrm{LEO}$ orbit 
Table 2 Measurement errors during the irradiation procedure

\begin{tabular}{lll}
\hline Error Source & Nominal Value & Error Range \\
\hline Sample Size & $45.26 \mathrm{~cm}^{2}$ & $\pm 1 \mathrm{~mm}$ per length measurement \\
Exposed Area & $42.32 \mathrm{~cm}^{2}$ & $\pm 0.958 \mathrm{~cm}^{2}$ \\
Current Stability & $100 \mathrm{nA}$ & $+0 \ldots+7 \mathrm{nA}$ \\
Timing & $0 \mathrm{~s}$ & $\pm 1 \mathrm{~s}$ \\
Fluence & $2.2 \times 10^{13} \mathrm{p}^{+} \mathrm{cm}^{-2}$ & $\pm 9.91 \times 10^{11} \mathrm{p}^{+} \mathrm{cm}^{-2}$ \\
\hline
\end{tabular}

under steady fluctuation around $100 \mathrm{nA}$ depending on gas flow in the proton source and probe voltage, extraction voltage and lens voltage, it is very unlikely that the samples were irradiated with great discrepancies to $100 \mathrm{nA}$ in average. The error range is set by the logged peak values.

\section{Results and Discussion}

\section{In-Situ Measurements}

The experiment was split into two phases. In the first phase, we irradiated the samples with $100 \mathrm{keV}$ protons with a current of $100 \mathrm{nA}$ for approximately $26.6 \mathrm{~min}$. In phase two, the proton irradiation was stopped. To estimate, if any detected effects only occur during proton irradiation, we observed the samples for another approximately $30 \mathrm{~min}$ under vacuum without radiation. Subsequently, the samples were removed from the irradiation chamber and placed in the airlock, which was flooded with nitrogen. This was to visibly confirm if any major changes of the surface occur after venting the airlock in terms of oxidation when the sample is removed from the airlock. Due to geometric reasons, it was not possible to apply LAMBDA at the airlock. Therefore, Fig. 5 displays the recorded behavior of phase 1 and 2, but not for the venting of the airlock.

In general, the reflectivity/brightness changes are relatively small and do not change by more than $2.5 \%$. Nonetheless, certain changes can be detected. In particular, the coated polyimide foil (panel b) of Fig. 5) showed a notable decrease in the reflected intensity during proton irradiation (phase 1). Furthermore, CFRP (panel a) showed a constant brightness during proton irradiation but an intensity drop was observed when the radiation was turned off. This indicates that the material might have been glowing due to surfaces charging. The metallic samples (Titanium c), Al 6082 d)) as well as PTFE, panel e) showed no significant radiation-induced changes in their reflectivity. AcktarBlack is a very absorptive material with an overall reflectivity $<2 \%$. Therefore, its reflectivity could not be observed since the signal is below the sensor sensitivity. 

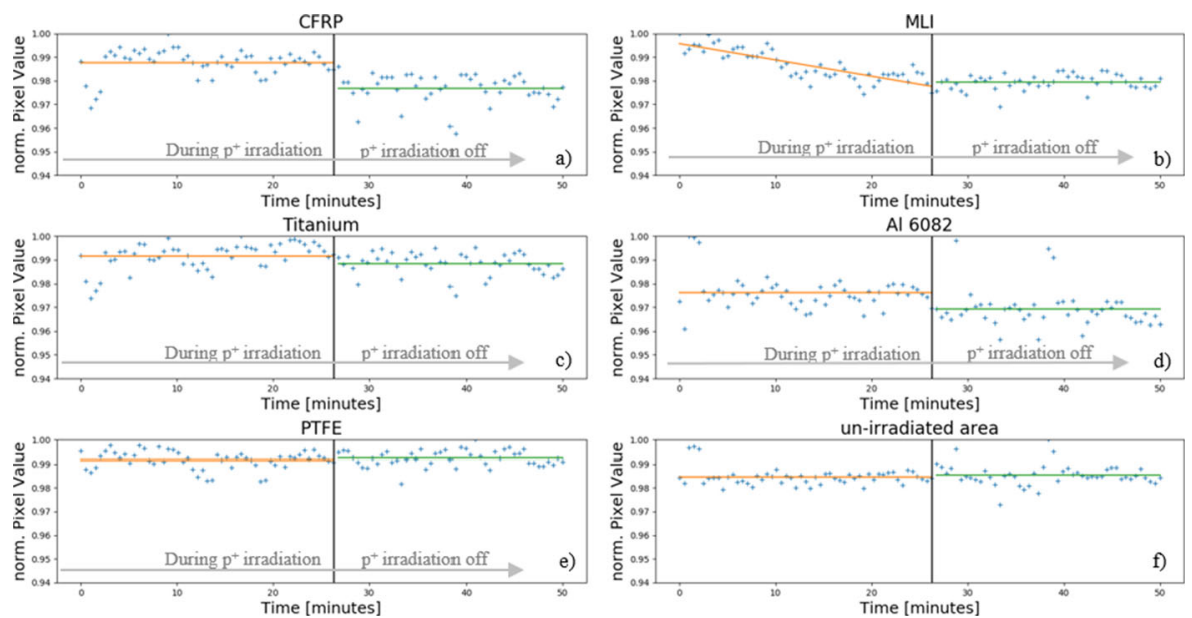

Fig. 5 Measurement results of LAMBDA

\section{Microscopic Measurements}

After releasing the target from the vacuum, it was immediately investigated with the digital microscope. We took measurements of the same samples and areas before and after the proton irradiation. At 200 times magnification, no structural damages due to protons were visible, see Fig. 6.

Afterwards, the samples were investigated with a SEM. This allows for the investigation with a higher resolution. Here, we took a set of reference samples and compared them to the irradiated sample set. We found that samples containing organic polymers (CFRP and PTFE) showed radiation induced surface modifications, while metallic samples, including coated polyimide foil, appeared unaffected by the proton irradiation. The detailed findings for PTFE and CFRP are as follows:

PTFE showed significant changes on the material surface structure, see Fig. 7. While the reference sample shows a plain structure with scratches and irregularities, the irradiated sample showed a very irregular surface which looks like melting patterns. As a thermoplastic with very low thermal conductivity and low radiative cooling ability due to its white appearance, melting seems to be a reasonable behavior.

CFRP was investigated for three conditions: changes in pure resin areas, changes in fiber dominated areas and changes in fiber-resin bonding, see Fig 8. Major changes were found in the pure resin areas (panels a) and b)), where abrasion of the surface has occurred. The reference surface is mostly plain with sharp grains and a few mountains. For the irradiated sample, almost no sharp grains are detectable; the surface appears smoother. Regarding the fibers, panel c) and d) they appear with better contrast for the irradiated sample. This indicates abrasion of the resin since the fibers are usually covered with a thin layer of resin. Furthermore, the reference shows resin conglomerates on the fiber surface while the irradiated sample shows a plainer surface with breaking patterns along the fiber. In the bonding area, panels e) and f), we 
Fig. 6 Microscope images (sample size $21 \times 21 \mathrm{~mm}^{2}$ ) of the samples before (left panel) and after (right panel) irradiation

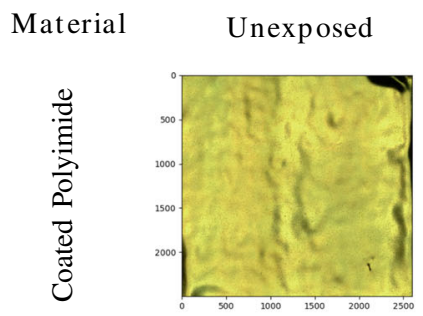

(a)

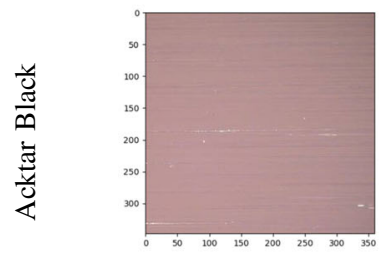

(c)

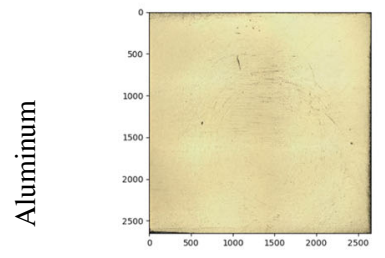

(e)

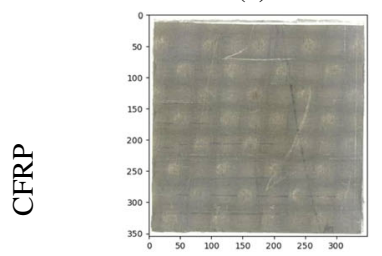

(g)

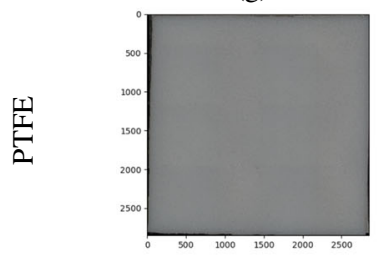

(i)

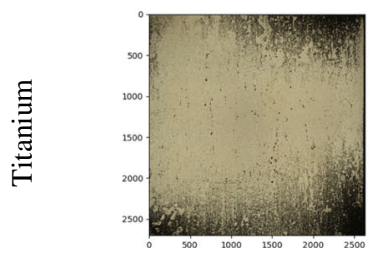

(k)
Exposed

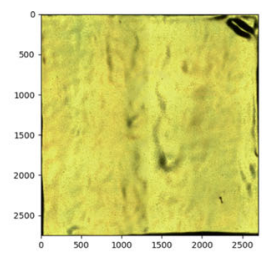

(b)

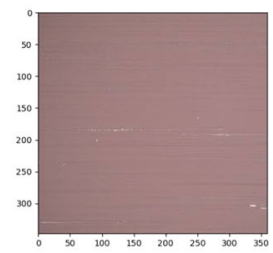

(d)

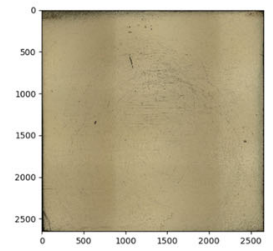

(f)

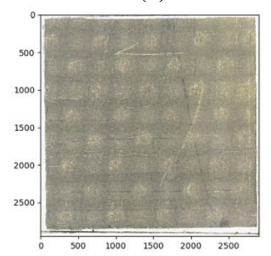

(h)

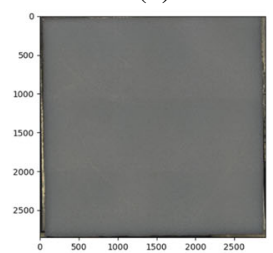

(j)

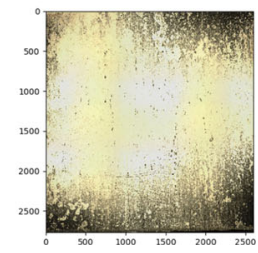

(1) 


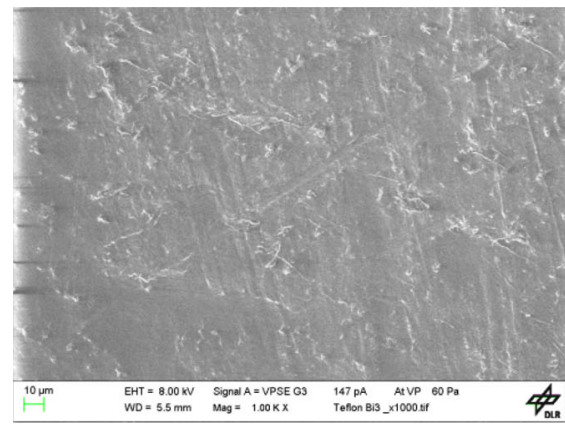

(a) PTFE unirradiated

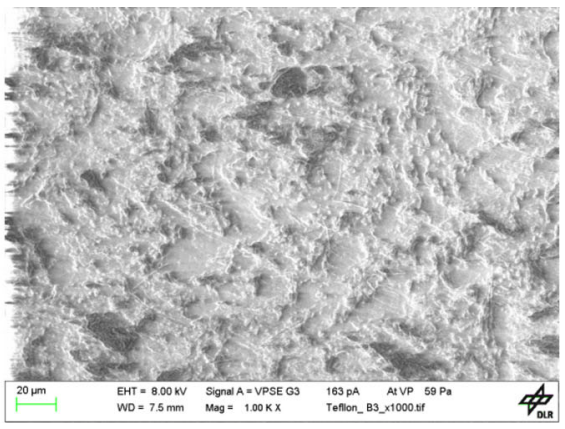

(b) PTFE irradiated

Fig. 7 Comparison of SEM image of the non-irradiated PTFE reference (a) and the proton irradiated sample (b)

can see fibers that are peaking out the resin for the irradiated sample. On the other side, for the references we see a blurry scheme of the fiber which indicates that it is still covered with a resin layer. We detected these results on several points on the sample surface.

\section{Spectral Analysis}

We also investigated the wavelength dependent direct reflectance of the samples at $30^{\circ}$ incidence/reflectance angle with the FTIR described in Section "Spectral Analysis", see Fig. 9. This allows for estimating possible wavelength depended spectral shifts due to proton related aging. As it turned out, none of the samples undergo any measurable color shifts in the whole wavelength range, except a slight variation of the $850 \mathrm{~nm}$ Aluminum feature. Nevertheless, we measured a change in reflectivity between the reference set and the irradiated sample set. Most significant is the change of reflectivity of aluminum (green) and titanium (orange). The reflectivity of the irradiated samples drops below the reflectivity of the unirradiated reference sample to approximately $60 \%$ and $75 \%$, respectively, of the initial value of the reference sample. Since both sets have been treated by the same polishing procedure, this can either be explained by manufacturing discrepancies or by re-oxidation of the surfaces after irradiation. Re-oxidation processes for metals, especially for aluminum, are expectable. Also, since there is no visible modification on the sample surface that could cause a less reflective behavior, such as tiny scratches, we considered reoxidation processes, which lead to a less reflective metal surface. This result would be consistent with the fact that we didn't measure significant reflectivity changes during proton irradiation.

Most interesting is the behavior of coated polyimide (brown). As we have measured before, its reflectivity has been reduced during the aging process. The purpose of coated polyimide as MLI layers is to protect a satellite from rising temperatures due to thermal radiation, but we observe a decay in reflectivity for the infrared range (wavenumbers smaller than $6000 \mathrm{~cm}^{-1}$ ). It drops from 0.9 (panel a)) to less than 0.75 


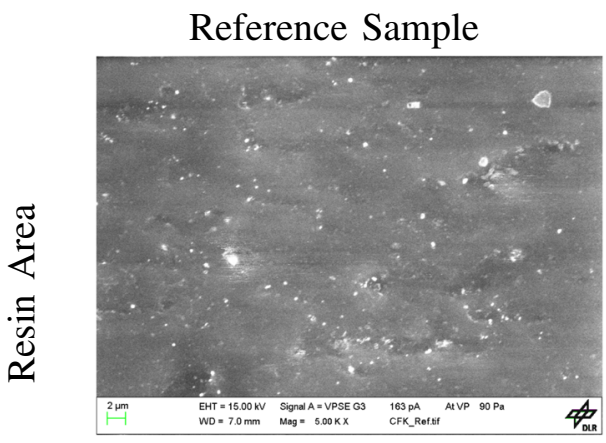

(a)

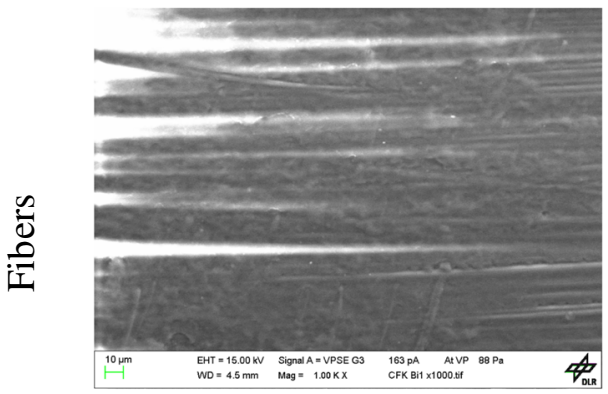

(c)

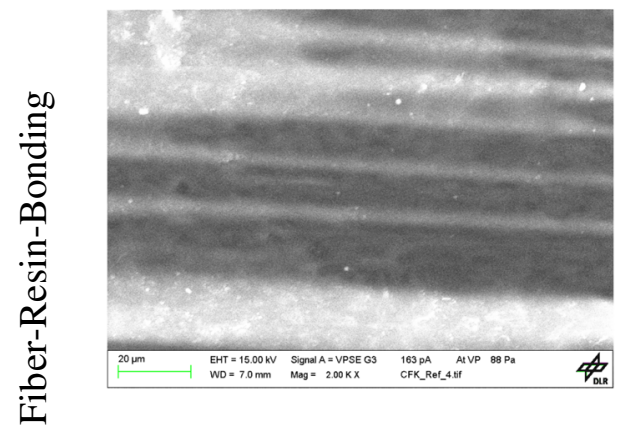

(e)

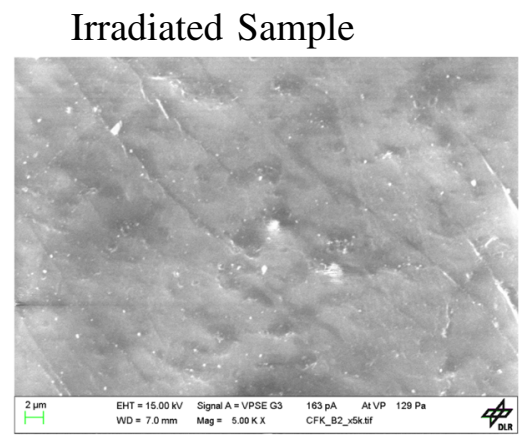

(b)

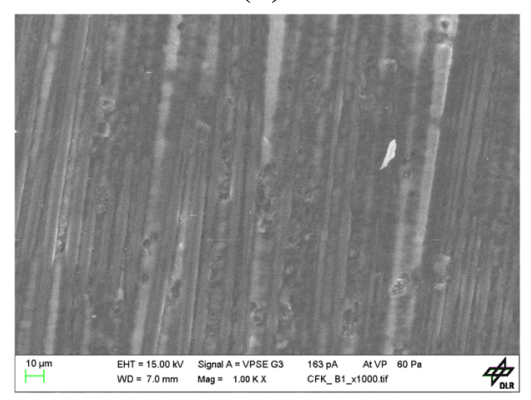

(d)

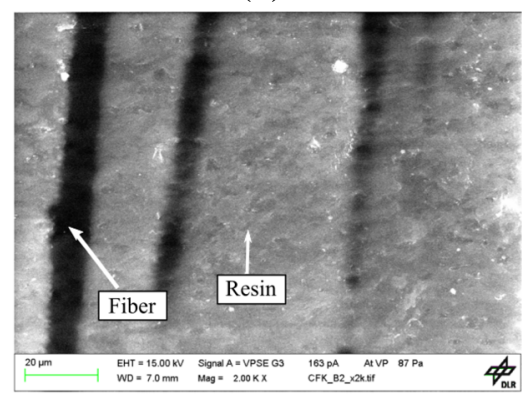

(f)

Fig. 8 SEM image of CFRP reference (left column) and sample (right column)

(panel b)). Panel c) shows that the foils's feature is affected by less than $10 \%$. In average, it lost about $20 \%$ of its reflectance. This confirms the result of LAMBDA.

On the other hand, polymer samples (light blue, red), including AcktarBlack (cyan), showed no variations in their spectral features (panel a) and b). CFRP and AcktarBlack are black and therefore very absorptive, while PTFE is a white but very diffusive material. That is why they don't show any significant specular reflections. The infra-red measurement shows a similar result like the visible with a peak for the 


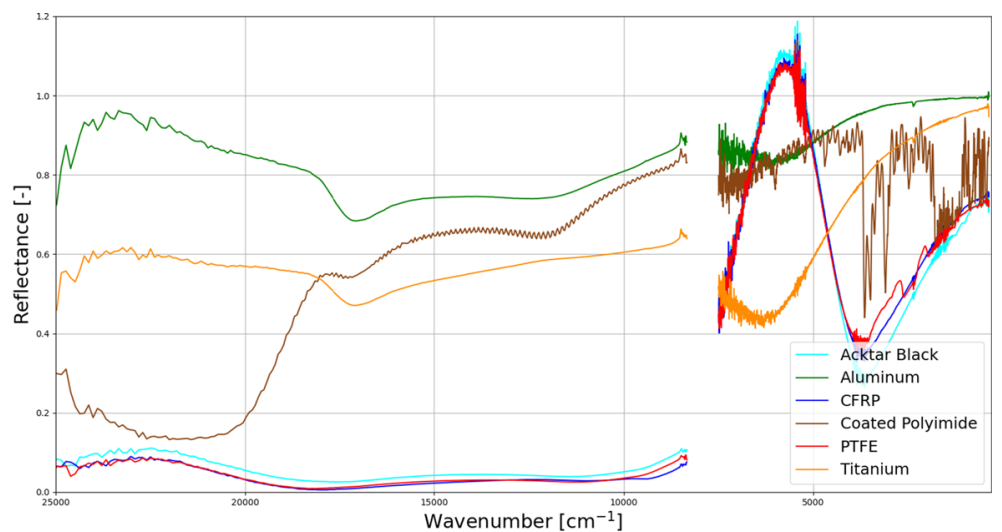

(a) Reflectivity of unirradiated reference samples at an angle of incidence of $30^{\circ}$

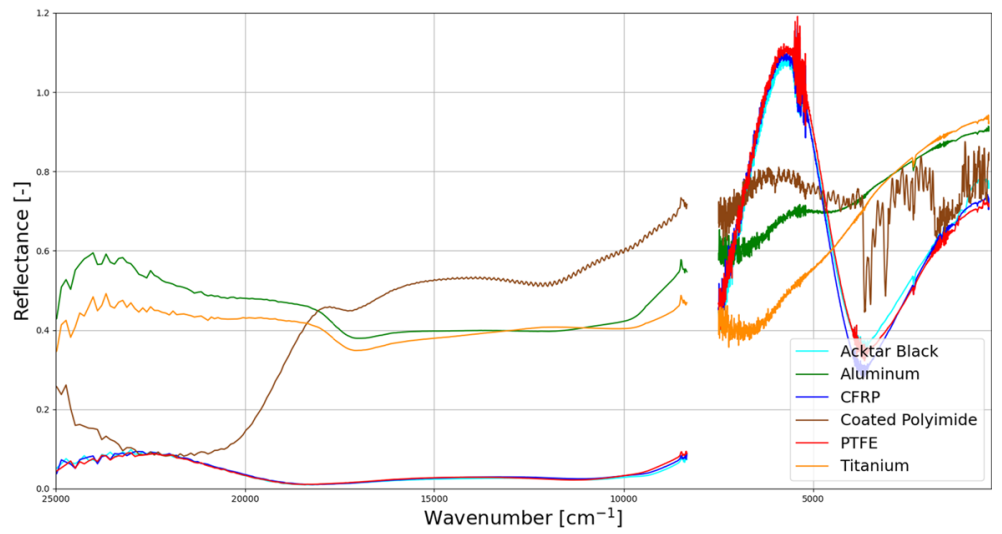

(b) Reflectivity of irradiated samples at an angle of incidence of $30^{\circ}$

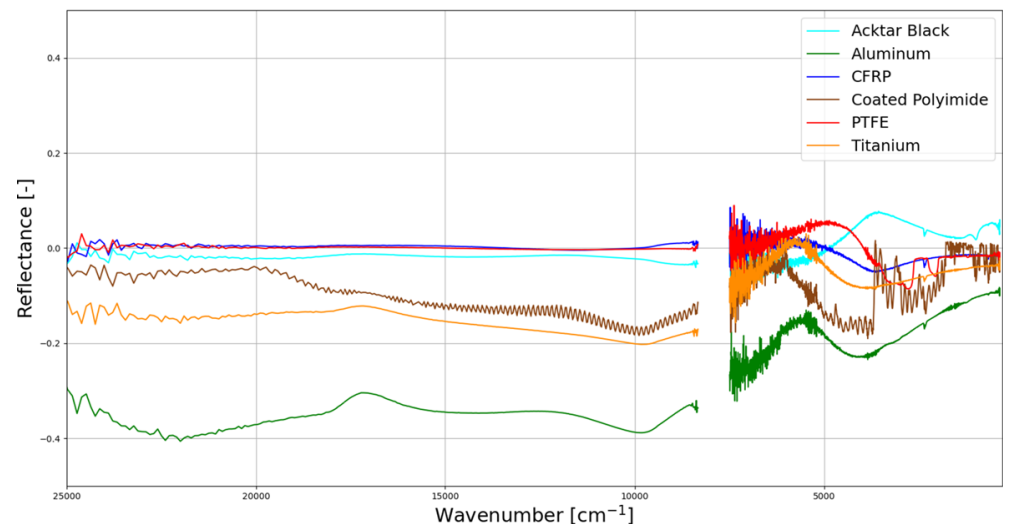

(c) Samples reflectivity normalized by reference reflectivity

Fig. 9 Comparison of reflection responses 
CFRP, AcktarBlack and PTFE around $5800 \mathrm{~cm}^{-1}$. Panel c) on the other hand indicates that there are changes which could not be detected by LAMBDA due to the low response of the samples. REM pictures, on the other hand, indicated changes in surface morphology.

\section{Conclusion}

We investigated the effects of low energetic proton irradiation equivalent to 800 $\mathrm{km}$ SSO LEO orbit on different types of material surfaces over a simulated time span of 100 years. A microscopic analysis of the irradiated samples reveals that materials containing organic polymers Polytetrafluoroethylene (PTFE) and carbon fiber reinforced plastic (CFRP) show significant changes in surface morphology. These changes can be directly detected using scanning electron microscopy SEM. As opposed to this, no major changes in the surface morphology were detected for metallic surfaces such as aluminum, titanium, AcktarBlack or ITO and aluminum coated polyimide as applied in multi-layer insulation (MLI). Changes in optical properties where detected for coated polyimide measured by LAMBDA and also affect the relative reflectivity as measured via FTIR spectroscopy. To solidify our conclusion, we intend to extend our irradiation experiments towards lower proton energies (e.g. 10 $\mathrm{keV})$ as well as to perform additional testing with low energetic electrons.

For further work, we intend to lower the proton energies based on a space environmental beta-model (e.g. GREEN model [26]) to provoke more protons interacting with the surface as well as applying electrons with an equivalent energy.

Acknowledgements The contributions from Dr. Patric Seefeldt, who supported our experiment during the corona lock-down, in the experimental procedure and in fruitful discussions of the gathered data are gratefully acknowledged. We also want to thank Samantha Siegert for proofreading the manuscript.

Funding Open Access funding enabled and organized by Projekt DEAL.

\section{Declarations}

Conflict of Interests The authors declare that they have no conflict of interest.

Open Access This article is licensed under a Creative Commons Attribution 4.0 International License, which permits use, sharing, adaptation, distribution and reproduction in any medium or format, as long as you give appropriate credit to the original author(s) and the source, provide a link to the Creative Commons licence, and indicate if changes were made. The images or other third party material in this article are included in the article's Creative Commons licence, unless indicated otherwise in a credit line to the material. If material is not included in the article's Creative Commons licence and your intended use is not permitted by statutory regulation or exceeds the permitted use, you will need to obtain permission directly from the copyright holder. To view a copy of this licence, visit http://creativecommons.org/licenses/by/4.0/.

\section{References}

1. Space debris mitigation guidelines. https://www.iadc-home.org/references/pdfview/id/75 
2. Liou, J.C., Anz-Meador, P.D., Opiela, J.N., Shoots, D.: History of on-orbit satellite fragmentations, 15th edn. Tech. rep., NASA Johnson Space Center; Houston, TX, United States (2018)

3. Newman, L., Hejduk, M., Frigm, R., Duncan, M.: Evolution and implementation of the NASA robotic conjunction assessment risk analysis concept of operations. In: Advanced Maui Optical and Space Surveillance Technologies Conference, p. E3 (2014)

4. Rossi, A., Valsecchi, G.B.: Collision risk against space debris in Earth orbits. Celest. Mech. Dyn. Astron. 95(1-4), 345-356 (2006). https://doi.org/10.1007/s10569-006-9028-7

5. Liou, J., Anilkumar, A., Bastida, B., Hanada, T., Krag, H., Lewis, H., Raj, M., Rao, M., Rossi, A., Sharma, R.: Stability of the future leo environment-an iadc comparison study. In: Proceedings of the 6th European Conference on Space Debris, vol. 723 (2013)

6. Liou, J.C., Matney, M., Vavrin, A., Manis, A., Gates, D.: Nasa odpo's large constellation study. Orbital Debris Quarterly News 22(3) (2018)

7. Hand, D., Tyler, D., Murali, S., Roggemann, M., Peterson N.: Real time polarization light curves for space debris and satellites (2010)

8. Dianetti, A.D., Crassidis, J.L.: Space Object material determination from polarized light curves. https://doi.org/10.2514/6.2019-0377. https://arc.aiaa.org/doi/abs/10.2514/6.2019-0377 (2019)

9. Hall, D., Hamada, K., Kelecy, T., Kervin, P.: Satellite surface characterization from non-resolved multi-band optical observations. In: Advanced Maui Optical and Space Surveillance Technologies Conference, p. 24 (2012)

10. Kurosaki, H., Yanagisawa, T., Nakajima, A.: Observation of light curves of space objects. In: Advanced Maui Optical and Space Surveillance Technologies Conference, p. E80 (2009)

11. Yanagisawa, T., Kurosaki, H.: Shape and motion estimate of leo debris using light curves. Adv. Space Res. 50(1), 136-145 (2012). https://doi.org/10.1016/j.asr.2012.03.021. https://www.sciencedirect. com/science/article/pii/S0273117712002013

12. Skurat, V., Barbashev, E., Dorofeev, Y., Nikiforov, A., Gorelova, M., Pertsyn, A.: Simulation of polymer film and surface behaviour in a space environment. Appl. Surface Sci. 92, 441446 (1996). https://doi.org/10.1016/0169-4332(95)00273-1. http://www.sciencedirect.com/science/ article/pii/0169433295002731. Proceedings of the Seventh International Conference on Solid Films and Surfaces

13. Dennison, J.R., Prebola, J., Evans, A., Fullmer, D., Hodges, J.L., Crider, D.H., Crews, D.S.: Comparison of flight and ground tests of environmental degradation of misse-6 suspecs materials. In: 11 th Spacecraft Charging Technology Conference (2010)

14. Engelhart, D.P., Cooper, R., Cowardin, H., Maxwell, J., Plis, E., Ferguson, D., Barton, D., Schiefer, S., Hoffmann R.: Space weathering experiments on spacecraft materials. In: 2017 Advanced maui optical and space surveillance technologies conference (AMOS). https://ntrs.nasa.gov/search.jsp? $\mathrm{R}=20170002370$ (2017)

15. Sznajder, M., Geppert, U., Dudek, M.: Degradation of metallic surfaces under space conditions, with particular emphasis on hydrogen recombination processes. Adv. Space Res. 27. https://doi.org/10.1016/j.asr.2015.03.032 (2015)

16. Horstmann, A., Kebschull, C., Müller, S., Gamper, E., Hesselbach, S., Soggeberg, K., Ben Larbi, M.K., Becker, M., Lorenz, J., Wiedemann, C., et al.: Survey of the current activities in the field of modeling the space debris environment at tu braunschweig. Aerospace 5(2), 37 (2018)

17. Scharring, S., Rodmann, J., Riede, W.: Network performance analysis of laser-optical tracking for space situational awareness in the lower earth orbit. In: 2019 Advanced Maui Optical and Space Surveillance Technologies Conference (AMOS) (2019)

18. Ganushkina, N.Y., Dandouras, I., Shprits, Y.Y., Cao, J.: Locations of boundaries of outer and inner radiation belts as observed by cluster and double star. Journal of Geophysical Research: Space Physics 116(A9). https://doi.org/10.1029/2010JA016376. https://agupubs.onlinelibrary.wiley.com/doi/abs/10. 1029/2010JA016376 (2011)

19. Nöldeke, C.M.: The space radiation environment. epubli. https://www.epubli.de/shop/buch/ The-Space-Radiation-Environment-Christoph-N (2017)

20. Renger, T., Sznajder, M., Geppert, U.: Experimental studies of low energy proton irradiation of thin vacuum deposited aluminum layers (2014)

21. Renger, T., Sznajder, M., Witzke, A., Geppert, U.: The complex irradiation facility at dlr-bremen. Journal of Materials Science and Engineering A 4. https://doi.org/10.1007/978-3-642-34907-2_34 (2014)

22. Sznajder, M., Geppert, U., Dudek, M.R.: Hydrogen blistering under extreme radiation conditions. npj Materials Degradation 2, 1-8 (2018) 
23. Sznajder, M., Seefeldt, P., Spröwitz, T., Renger, T., Kang, J., Bryant, R., Wilkie, W.: Solar sail propulsion limitations due to hydrogen blistering. Advances in Space Research. https://doi.org/10.1016/j. asr.2020.06.034. http://www.sciencedirect.com/science/article/pii/S0273117720304543 (2020)

24. Theiss, W.: Scout, wtheiss hardware and software, Tucson, USA. http://www.wtheiss.com/. V5.06 (2021)

25. Heynderickx, D., Quaghebeur, B., Evans, H.D.R.: The ESA space environment information system (SPENVIS). In: IAF abstracts, 34th COSPAR Scientific Assembly, p. 475 (2002)

26. Sicard, A., Boscher, D., Bourdarie, S., Lazaro, D., Standarovski, D., Ecoffet, R.: GREEN: the new Global Radiation Earth ENvironment model (beta version). Annales Geophysicae 36, 953-967 (2018). https://doi.org/10.5194/angeo-36-953-2018. https://hal.archives-ouvertes.fr/hal-02126965

Publisher's Note Springer Nature remains neutral with regard to jurisdictional claims in published maps and institutional affiliations. 